

\title{
CAMBIOS DE LA CONDUCTIVIDAD ELÉCTRICA ASOCIADOS AL TRATAMIENTO MAGNÉTICO DEL AGUA
}

\author{
Jaime Ernesto Díaz Ortíz* \\ María Victoria Carbonell Padrino** \\ Elvira Martínez Ramírez***
}

* Ingeniero Agrícola Ph.D. Profesor Titular Escuela de Ingeniería de Recursos Maturales y del Ambiente, Universidad del Valle

** Ingeniera Agrónoma Ph.D. Profesora Universidad Politécnica de Madrid

*** Ciencias Químicas Ph.D. Profesora Universidad Politécnica de Madrid

\section{RESUMEN}

El experimento estudia el efecto del tratamiento magnético en la conductividad eléctrica del agua destilada. El agua se sometió a diferentes tiempos de tratamiento magnético $(30,60,120)$ minutos. Los resultados mostraron diferencias significativas superiores al 95\%, entre los distintos tratamientos y también frente al control. Se encontró que el agua destilada incrementó la conductividad eléctrica de la misma, de una forma lineal, presentando coeficientes lineales $r^{2}$ $=0.85$ o superiores. Este experimento forma parte de la tesis doctoral "Estudio de factores que afectan la evaporación del agua en el suelo", 
desarrollada en la Universidad Politécnica de Madrid.

\section{ABSTRACT}

This experiment studies the effect that the diference magnetic treatment carries out about the electric conductivity of the distilled water, using three types of magnetic treatments $(30,60,120$ minutes). Differences were detected statistically significant among the different treatments and also between the treatments and the control with values above 95\%. The rehearsals carried out in the experiment showed that in the distilled water the increase of the time of magnetically inceased the electric conductivity in a lineal way with values of coefficient of lineal regression above 0.85 . The experiment was make at Universidad Politécnica de Madrid.

\section{INTRODUCCIÓN}

Cuando el agua pasa a través de un campo magnético de dirección e intensidad apropiada, sus moléculas son orientadas y se producen cambios en las propiedades físico-químicas.

Estudios realizados en laboratorio acerca del tratamiento magnético del agua, muestran cambios en la solubilidad de los compuestos y forma de los cristales. Cuando el agua se trata magnéticamente, el carbonato cálcico contenido cambia su forma de cristalización de calcita a aragonita. Tradicionalmente la principal aplicación del tratamiento magnético del agua ha consistido en prevenir la formación de incrustaciones en tuberías y calderas. Tratamientos diferentes a los químicos fueron primero propuestos para controlar la formación de incrustaciones en el siglo diecinueve; las primeras patentes para el tratamiento del agua empleando un campo magnético fueron desarrolladas por Porter (1865) y Hay (1873) en Estados Unidos. Actualmente muchos de esos equipos se encuentran disponibles comercialmente y tienen una efectividad alta para el control de las incrustaciones en sistemas donde se utiliza agua, ayudando además a controlar la corrosión. (Limpert and Raber, 1985). Más recientemente nuevas aplicaciones del tratamiento magnético del agua se están enfocando a la búsqueda de soluciones de problemas técnicos como alternativas de producción limpia. Ejemplo de esto puede ser el control de algas por tratamiento magnético en el agua de las piscinas o en las tuberías de riego por goteo.

Gonet (1985) observó que la exposición del agua tridestilada a campos magnéticos variables de $120 \mathrm{mT}$ y $800 \mathrm{mT}(1 \mathrm{kHz})$, originaban cambios en la constante dieléctrica del $1 \%$.

Martin A. et al (1996) determinaron que la cantidad de productos bactericidas se disminuía en un $60 \%$ y de algicidas hasta un $100 \%$, en sistemas de depuración de aguas cuando se realizaban tratamientos magnéticos.

Busch et al (1997), indicaron que los efectos magnetohidrodinámicos producidos por los dispositivos magnéticos, ayudaban a controlar la formación de depósitos calcáreos en sistemas que utilizan agua.

También la reducción de incrustaciones de carbonato de calcio fueron observados por Higashitani et al (1995) y Parson et al., (1997) utilizando métodos de absorbancia en el ultravioleta visible, difracción de rayos $x y$ mediciones de amplitud de la fuerza microscópica repulsivas entre las partículas.

Estudios realizados por Carbonell et al (1996) y Martinez et al (1999), revelaron modificaciones en algunas propiedades físico-químicas del agua tratada magnéticamente tales como solubilidad y modificaciones del pH. En este artículo se evalúa el efecto que el tratamiento magnetohdrodinámico del agua destilada ejerce en la conductividad eléctrica. 


\section{MATERIALES Y MÉTODOS}

El experimento se desarrolló en condiciones de laboratorio, utilizando agua destilada. La temperatura de la habitación fue de $20^{\circ} \pm 2^{\circ} \mathrm{C}$.

El agua fue tratada magnéticamente por método dinámico, haciéndola recircular en una tubería de $30 \mathrm{~m}$ de longitud y diámetro interno de $19 \mathrm{~mm}$ adoptando forma de dobla espira. Seis baterías magnéticas se colocaron a lo largo del tubo y cada batería se componía de dos imanes de 1.25 $\mathrm{mT}$ enfrentados en polos iguales. Las líneas del campo magnético se encontraban perpendicularmente a la dirección del flujo.

El agua se movía en la tubería por medio de una bomba con una potencia de $55 \mathrm{~W}$. La velocidad de circulación del agua en la tubería fue variable, realizando 3 ensayos. Para el primero de los ensayos se realizaron cuatro repeticiones haciendo funcionar el equipo de magnetización de agua a 1200 revoluciones por minuto. En el segundo ensayo se modificó la velocidad del equipo a 1850 revoluciones por minuto y se realizaron 2 repeticiones.

El tercer ensayo (blanco) tuvo dos repeticiones, fijando la velocidad de circulación del agua dentro de la tubería en 1200 revoluciones por minuto, pero sin exponer el agua a ningún campo magnético $(\mathrm{I}=0 \mathrm{mT})$.

El agua fue expuesta a tres tiempos de magnetización:

$\mathrm{T}_{1}=$ recirculación por 30 minutos

$\mathrm{T}_{2}=$ recirculación por 60 minutos

$\mathrm{T}_{3}=$ recirculación por 120 minutos

Agua destilada sin ningún tratamiento fue usada como control (C).
El equipo era vaciado totalmente después que el agua fue tratada magnéticamente en cada tratamiento. Al iniciar cada tratamiento se recogía una muestra de agua magnetizada y a continuación se colocaba en 12 recipientes que eran cerrados herméticamente. Diariamente se escogían 3 recipientes por tratamiento, se determinaba la conductividad eléctrica y se realizaba un promedio de las lecturas obtenidas. Una vez que se efectuaban las lecturas, las muestras de estos recipientes eran desechadas. El tiempo de duración de cada uno de los ensayos fue de cuatro días y el número de ensayos fue de 6.

Las medidas de la conductividad eléctrica expresadas en $m \mu . \mathrm{cm}^{-1}$ se realizaron con un conductímetro digital marca Orion modelo 150, que corrige automáticamente los cambios de temperatura de las muestras, tomando como referencia una temperatura estándar de $25^{\circ} \mathrm{C}$.

\section{RESULTADOS Y DISCUSIÓN}

Los resultados de las medias para los valores de la conductividad eléctrica en el agua destilada tratada magnéticamente durante 30, 60, 120 minutos y el control, se presentan en las tablas del 1 al 6 . Los resultados de los ensayos en blanco se presentan en las tablas 7 y 8 . La tabla 9 presenta los valores de probabilidad obtenidos según diferentes test aplicados, los resultados de las regresiones lineales obtenidas y el coeficiente de regresión lineal. Las diferencias detectadas entre los distintos tratamientos entre sí y frente al control se establecieron para niveles de probabilidad del $95 \%$ en los diferentes test aplicados.

Los resultados de las tablas 1 al 6 muestran que el tratamiento magnetohidrodinámico del agua destilada incrementa significativamente la conductividad eléctrica. El valor medio de la conductividad eléctrica se incrementa con el tiempo de recirculación del agua dentro del equipo magnético. 
El análisis de varianza Anova mostró una diferencia extremadamente significativa $(p=0.0001)$ entre las medias de los tratamientos para todas las repeticiones de los ensayos 1 y 2 . La variación de la velocidad en ambos ensayos sirvió para descartar que la velocidad de la bomba influyera decisivamente en los cambios de la conductividad eléctrica del agua.

La aplicación del test paramétrico múltiple TukeyKramer, mostró que se presentaron diferencias significativas entre todos los tratamientos $\left(T_{1}, T_{2} Y\right.$ $T_{3}$ ) y el control (C), así como también entre los tratamientos entre sí. El test paramétrico simple Dunnett, mostró diferencias muy significativas entre cada uno de los tratamientos y el control.

Las repeticiones del ensayo 3 (blanco) mostraron diferencias significativas en ambas cuando se realizó el análisis de varianza Anova, pero al aplicar los test de Tukey-Kramer y Dunnett no se presentaron diferencias entre los tratamientos y el control.

El análisis de regresión lineal mostró coeficientes de regresión altos, indicando que existe una correspondencia adecuada entre el tiempo de duración del tratamiento magnético del agua destilada y el incremento de la conductividad eléctrica.

La figura 1 muestra la variación de la conductividad eléctrica del agua destilada con el tiempo de magnetización para una de las repeticiones. Se observa un incremento de la conductividad eléctrica al aumentar el tiempo de recirculación de la misma en el equipo de magnetización.

Al analizar las ecuaciones de regresión obtenidas en los diferentes ensayos se observa que los coeficientes de regresión lineal son superiores a 0.87 , indicando una relación bastante aceptable entre el tiempo de magnetización del agua y la conductividad eléctrica.
Por el contrario los resultados obtenidos en las repeticiones del ensayo 3 (blanco), muestran escasa correspondencia entre el tratamiento magnético del agua y el incremento de la conductividad eléctrica.

Este incremento de la conductividad eléctrica con el tiempo de magnetización está de acuerdo con los resultados obtenidos en las torres de enfriamiento, unidades de impulsión de agua e intercambiadores de calor. Los reportes que presenta la revisión bibliográfica acerca del funcionamiento de estos equipos indican que la recirculación del agua aumenta la conductividad eléctrica del flujo en circulación. Para conseguir mejores resultados Busch et al (1997) recomiendan que el campo magnético debe ser orientado normalmente en la dirección del flujo del fluido.

Ensayo 1

Repetición 1

Tabla 1 Conductividad eléctrica del agua destilada $\left(\mu \mathrm{S} . \mathrm{cm}^{-1}\right)$

\begin{tabular}{|c|c|c|c|c|}
\hline TRATAMIEMTO & DÍA1 & DÍA2 & DÍA3 & DÍA4 \\
\hline$C$ & 9.3 & 8.7 & 8.5 & 8.6 \\
\hline$T_{1}$ & 11.2 & 11.0 & 10.3 & 10.4 \\
\hline$T_{2}$ & 12.6 & 11.9 & 11.6 & 11.3 \\
\hline$T_{3}$ & 17.2 & 16.5 & 16.8 & 16.7 \\
\hline
\end{tabular}

Repetición 2

Tabla 2 Conductividad eléctrica del agua destilada $\left(\mu\right.$ S.cm $\left.{ }^{-1}\right)$

\begin{tabular}{|c|c|c|c|c|}
\hline TRATAMIEMTO & DÍA1 & DÍA2 & DÍA3 & DÍA4 \\
\hline$C$ & 5.1 & 5.1 & 5.0 & 4.7 \\
\hline$T_{1}$ & 5.6 & 5.0 & 4.9 & 4.8 \\
\hline$T_{2}$ & 7.5 & 7.4 & 7.4 & 7.5 \\
\hline$T_{3}$ & 11.6 & 12.8 & 14.0 & 13.9 \\
\hline
\end{tabular}


Repetición 3

Tabla 3 Conductividad eléctrica del agua destilada $\left(\mu \mathrm{S} . \mathrm{cm}^{-1}\right)$

\begin{tabular}{|c|c|c|c|c|}
\hline TRATAMIEMTO & DÍA1 & DÍA2 & DÍA3 & DÍA4 \\
\hline$C$ & 4.3 & 4.3 & 3.8 & 4.0 \\
\hline$T_{1}$ & 6.2 & 5.5 & 5.3 & - \\
\hline$T_{2}$ & 7.4 & 7.1 & 7.3 & 6.6 \\
\hline$T_{3}$ & 16.5 & 15.5 & 15.4 & 15.1 \\
\hline
\end{tabular}

Repetición 4

Tabla 4 Conductividad eléctrica del agua destilada $\left(\mu \mathrm{S} . \mathrm{cm}^{-1}\right)$

\begin{tabular}{|c|c|c|c|c|}
\hline TRATAMIEMTO & DIA1 & DIA2 & DÍA3 & DÍA4 \\
\hline$C$ & 3.8 & 3.5 & 3.8 & 3.9 \\
\hline$T_{1}$ & 4.9 & 4.1 & 3.7 & 4.0 \\
\hline$T_{2}$ & 5.6 & 4.7 & 5.2 & 5.2 \\
\hline$T_{3}$ & 9.4 & 8.9 & 9.0 & 9.4 \\
\hline
\end{tabular}

Intensidad de magnetización $=125 \mathrm{mT}$. Velocidad de bomba $=1200 \mathrm{rpm}$.

C: control; $\mathrm{T}_{1}: 30$ minutos; $\mathrm{T}_{2}: 60$ minutos; $T_{3}: 120$ minutos

Ensayo 2

Repetición 1

Tabla 5 Conductividad eléctrica del agua destilada $\left(\mu \mathrm{S} . \mathrm{cm}^{-1}\right)$

\begin{tabular}{|c|c|c|c|c|}
\hline TRATAMIEMTO & DÍA1 & DIA2 & DÍA3 & DÍA4 \\
\hline$C$ & 4.6 & 4.4 & 4.9 & 5.2 \\
\hline$T_{1}$ & 7.7 & 6.9 & 6.9 & 6.9 \\
\hline$T_{2}$ & 12.0 & 12.3 & 12.7 & 12.0 \\
\hline$T_{3}$ & 19.0 & 19.2 & 18.8 & 19.2 \\
\hline
\end{tabular}

Repetición 2

Tabla 6 Conductividad eléctrica del agua destilada $\left(\mu \mathrm{S} . \mathrm{cm}^{-1}\right)$

\begin{tabular}{|c|c|c|c|c|}
\hline TRATAMIEMTO & DÍA1 & DÍA2 & DÍA3 & DÍA4 \\
\hline C & 7.7 & 7.9 & 8.0 & 8.3 \\
\hline$T_{1}$ & 8.8 & 7.7 & 7.8 & 8.2 \\
\cline { 1 - 1 }$T_{2}$ & 11.1 & 10.8 & 10.7 & 10.9 \\
\hline$T_{3}$ & 11.4 & 11.3 & 11.5 & 12.0 \\
\hline
\end{tabular}

Intensidad de magnetización $=125 \mathrm{mT}$.

Velocidad de bomba $=1850 \mathrm{rpm}$.

C: control; $T_{1}: 30$ minutos; $T_{2}: 60$ minutos;

$\mathrm{T}_{3}: 120$ minutos

Ensayo 3

Repetición 1

Tabla 7 Conductividad eléctrica del agua destilada $\left(\mu \mathrm{S} . \mathrm{cm}^{-1}\right)$

Blanco 1

\begin{tabular}{|c|c|c|c|c|}
\hline TRATAMIEMTO & DÍA1 & DÍA2 & DÍA3 & DÍA4 \\
\hline$C$ & 3.8 & 3.5 & 4.3 & 3.4 \\
\hline$T_{1}$ & 4.6 & 4.2 & 6.7 & 6.3 \\
\hline$T_{2}$ & 4.5 & 4.1 & 4.1 & 3.9 \\
\hline$T_{3}$ & 4.9 & 3.9 & 6.9 & 6.5 \\
\hline
\end{tabular}

Repetición 2

Tabla 8 Conductividad eléctrica del agua destilada $\left(\mu \mathrm{S} . \mathrm{cm}^{-1}\right)$

Blanco 2

\begin{tabular}{|c|c|c|c|c|}
\hline TRATAMIEMTO & DÍA1 & DÍA2 & DíA3 & DÍA4 \\
\hline$C$ & 3.6 & 4.0 & 3.6 & 3.8 \\
\hline$T_{1}$ & 3.8 & 4.2 & 4.1 & 4.1 \\
\hline$T_{2}$ & 4.2 & 3.9 & 3.9 & 3.6 \\
\hline$T_{3}$ & 4.2 & 4.0 & 4.1 & 4.2 \\
\hline
\end{tabular}

Intensidad de magnetización $=125 \mathrm{mT}$.

Velocidad de bomba $=1850 \mathrm{rpm}$.

C: control; $T_{1}: 30$ minutos; $T_{2}: 60$ minutos;

$T_{3}: 120$ minutos 


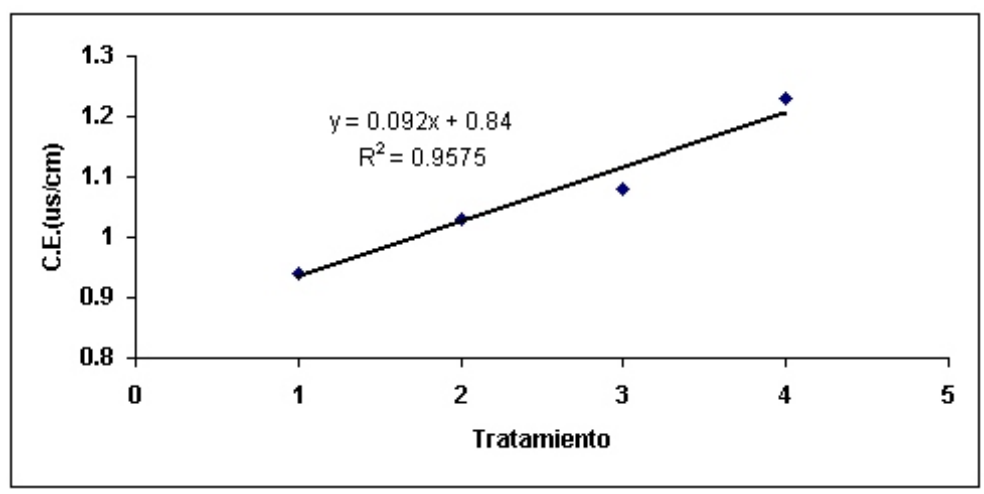

Figura 1. Variación de la conductividad eléctrica del agua $\left(\mu 5 . \mathrm{cm}^{-1}\right)$ con el tratamiento magnético. La línea de tendencia representa los valores promedios del ensayo y se presenta el valor de $r^{2}$ y la ecuación de la regresión.

\begin{tabular}{|c|c|c|c|c|c|c|}
\hline \multirow{37}{*}{$\begin{array}{l}\text { Tabla } 9 \\
\text { Análisis Estadístico }\end{array}$} & \multirow[t]{3}{*}{ Ensayo } & \multirow[t]{3}{*}{ Repetición } & \multicolumn{4}{|c|}{ Probabilidad } \\
\hline & & & \multicolumn{2}{|l|}{ Test } & & $R^{2}$ \\
\hline & & & Anova & Tukey & Dunnett & \\
\hline & \multirow[t]{22}{*}{1} & \multirow[t]{6}{*}{1} & \multirow[t]{6}{*}{$0,0001^{* *}$} & $\mathrm{C}-\mathrm{T}_{1}{ }^{\cdots \cdots}$ & $\mathrm{C}-\mathrm{T} 1^{* *}$ & \multirow[t]{6}{*}{0,96} \\
\hline & & & & C-T2"** & $\mathrm{C}-\mathrm{T} 2^{* *}$ & \\
\hline & & & & $\mathrm{C}-\mathrm{T}^{* * * *}$ & C-T3** & \\
\hline & & & & $\mathrm{T}_{1-\mathrm{T} 2^{*}}$ & & \\
\hline & & & & T1-T3*** & & \\
\hline & & & & T2-T3** & & \\
\hline & & \multirow[t]{5}{*}{2} & \multirow[t]{5}{*}{$0,0001^{* * *}$} & $\mathrm{C}-\mathrm{T} 2^{* * *}$ & & \multirow[t]{5}{*}{0,88} \\
\hline & & & & $\mathrm{C}-\mathrm{T}^{* * *}$ & C-T2** & \\
\hline & & & & T1-T2*** & C-T3** & \\
\hline & & & & T1-T3*** & & \\
\hline & & & & T2-T3** & & \\
\hline & & \multirow[t]{6}{*}{3} & \multirow[t]{6}{*}{$0,0001^{* \cdots}$} & C-T1 ${ }^{* \cdots}$ & C-T1 $1^{* *}$ & \multirow[t]{6}{*}{0,92} \\
\hline & & & & $\mathrm{C}-\mathrm{T} 2^{* * *}$ & C-T2** & \\
\hline & & & & $\mathrm{C}-\mathrm{T} 3^{* * *}$ & C-T3** & \\
\hline & & & & T1-T2* & & \\
\hline & & & & T1-T3*** & & \\
\hline & & & & T2-T3** & & \\
\hline & & \multirow[t]{5}{*}{4} & \multirow[t]{5}{*}{$0,0001^{* * *}$} & C-T2"** & $\mathrm{C}-\mathrm{T}^{* *}$ & \multirow[t]{5}{*}{0,88} \\
\hline & & & & C-T3"** & & \\
\hline & & & & T1-T2* & & \\
\hline & & & & T1-T3"* & & \\
\hline & & & & T2-T3"* & & \\
\hline & \multirow[t]{10}{*}{2} & \multirow[t]{6}{*}{1} & \multirow[t]{6}{*}{$0,0001^{* * *}$} & C-T1 ${ }^{\cdots}$ & C-T1"* & \multirow[t]{6}{*}{1,0} \\
\hline & & & & $\mathrm{C}-\mathrm{T} 2^{* * *}$ & $\mathrm{C}-\mathrm{T} 2^{* *}$ & \\
\hline & & & & C-T3** & & \\
\hline & & & & T1-T2"** & & \\
\hline & & & & T1-T3** & & \\
\hline & & & & T2-T3** & & \\
\hline & & \multirow[t]{4}{*}{2} & \multirow[t]{4}{*}{$0,0001^{* * *}$} & $C-T 2^{* * *}$ & & \multirow[t]{4}{*}{0,87} \\
\hline & & & & C-T3"** & C-T2* & \\
\hline & & & & T1-T2"** & C-T3** & \\
\hline & & & & T3-T3*** & & \\
\hline & \multirow[t]{2}{*}{3} & 1 & $0,0468^{\cdots \cdots}$ & n.s & n.s & 0,34 \\
\hline & & 2 & $0,0025^{* \cdots}$ & n.s & n.s & 0,57 \\
\hline
\end{tabular}




\section{CONCLUSIONES}

La conductividad eléctrica del agua destilada aumenta cuando el agua es tratada magnéticamente.

El incremento de la conductividad eléctrica tiene relación con el incremento del tiempo de circulación del agua en el equipo magnético.

En todas las repeticiones de los ensayos realizados, el incremento de la conductividad eléctrica del agua muestra un coeficiente de regresión lineal superior a 0.85 , indicando una relación bastante aceptable entre el tiempo de tratamiento magnético del agua y el incremento de la conductividad eléctrica.

Las dos repeticiones realizados en el ensayo 3 (blanco), señalan poca representatividad estadística en los resultados obtenidos, confirmando la influencia del tratamiento magnético del agua en la variación de la conductividad eléctrica.

\section{BIBLIOGRAFÍA}

Busch, W. K; Busch, M. A. 1997. Laboratory studies on magnetic water treatment and their relationship to a possible mechanism for scale reduction. Desalination. 109, 131-148.

Carbonell, M. V.; Martinez, E. 1996 Estudio de la solubilidad de distintos compuestos en agua imantada. Química e Industria 43, 21 -23.

Gonet, B. 1985. Influence of constant magnetic fields on certain physiochemical propierties of water. Bioelectromagnetics 6: 169-175.

Hay, A. T. 1873. U.S. Patent Mo140196, June 24.

Higashitani, K.; Iseri, H.; Okuhara, K.; Kage, A.; Hatade, S. 1995. Magnetic effects on Zeta Potential and Difusivity on Monmagnetic Colloidal
Particles. Journal of Colloidal and Interface Science $172,383-388$.

Limpert, G.J.C.; Raber, J. L. 1985. Test of MonChemical scale control device in a Once-Through System. Corrosion. Mational Association of Corrosion Engineers, Boston, Massachusets. Mo 250.

Martín, A.; Bejar Padilla, Mª L.; Sanchez, T. 1996. Estudio del magnetismo en aguas de piscinas. 61-62. Tecnoambiente.

Parsons, S.; Wang, B.; Judd, S.; Stephenson; T. 1997. Magnetic treatment of calcium carbonate. scale effect on $\mathrm{pH}$ control. Water Research, Vol 31, MO2: $339-342$.

Porter, A.F. 1865. U. S. Patent N050774, Octuber 31. 